\title{
Toward the Design of New Suitable Materials for Solar Water Splitting Using Density Functional Theory
}

\author{
Moussab Harb*๑ and Luigi Cavallo ${ }^{\odot}$ \\ KAUST Catalysis Center, Physical Sciences and Engineering Division, King Abdullah University of Science and Technology \\ (KAUST), Thuwal 23955-6900, Saudi Arabia
}

\section{Supporting Information}

\begin{abstract}
We report key results of a systematic computational investigation using density functional theory along with the two standard Perdew-Burke-Ernzerhof and hybrid Heyd-ScuseriaErnzerhof (HSE06) exchange-correlation formalisms on essential fundamental parameters for solar energy conversion of a series of large, medium, and small selected (covalent, binary, and ternary) materials widely utilized in fuel cells, photocatalysis, optoelectronics, photovoltaics, and dye-sensitized solar devices such as $\mathrm{BN}$, AlN, C, $\mathrm{ZrO}_{2}, \mathrm{Na}_{2} \mathrm{Ta}_{4} \mathrm{O}_{11}, \mathrm{Bi}_{4} \mathrm{Ti}_{3} \mathrm{O}_{12}, \mathrm{ZnS}, \mathrm{GaN}, \mathrm{SrTiO}_{3}, \mathrm{TiO}_{2}$, $\mathrm{Bi}_{12} \mathrm{TiO}_{20}, \mathrm{SiC}, \mathrm{WO}_{3}, \mathrm{TaON}, \mathrm{ZnSe}, \mathrm{BiVO}_{4}, \mathrm{CuNbO}_{3}, \mathrm{CdS}, \mathrm{AlP}$, $\mathrm{ZnTe}, \mathrm{GaP}, \mathrm{Cu}_{2} \mathrm{O}, \mathrm{AlAs}, \mathrm{Ta}_{3} \mathrm{~N}_{5}$, BP, CdSe, $\mathrm{SnWO}_{4}, \mathrm{GaAs}, \mathrm{CdTe}$, and $\mathrm{Si}$. Our calculations highlight that the optoelectronic and redox parameters computed with HSE06 reproduce with very

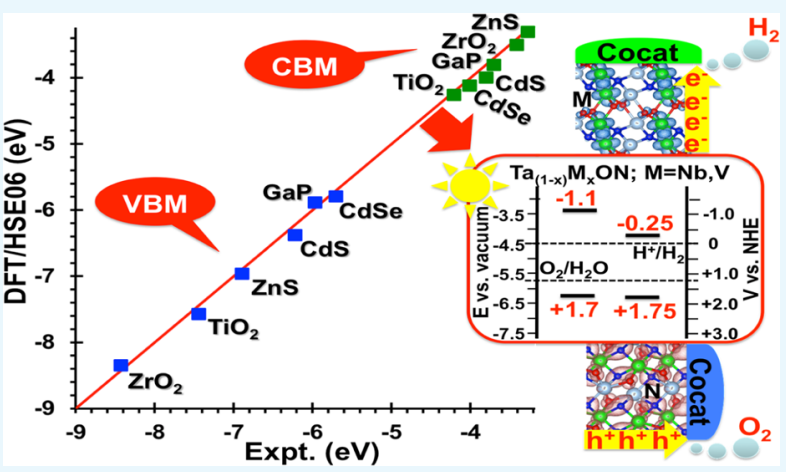
good accuracy the experimental results, thanks to precise electronic structure calculations. Applying this first-principle quantum methodology led us to provide a rational design of new suitable solid solution materials for visible light-driven photochemical water splitting. This valuable computational tool will be applied to predict promising candidates to be experimentally prepared and tested for solar-to-chemical energy conversion.
\end{abstract}

\section{INTRODUCTION}

The design of new materials with particular fundamental features in relation with solar light absorption, dissociation ability of photogenerated exciton, transport of charge carriers, and their ability to be driven for water redox reactions is of major importance for converting solar energy into clean hydrogen from water splitting. ${ }^{1-3}$

Although the fabrication of highly crystalline material with a very minimal number of defects is essential, the design of a suitable photocatalyst for good solar-driven water splitting needs at least these four additional challenging fundamental parameters to be present: (a) specific band gap energy between 2.0 and $2.5 \mathrm{eV}$ for good solar photon absorption in the visible region; (b) high macroscopic dielectric constant (greater than 10) together with low exciton binding energy $(<25 \mathrm{meV})$ for the good dissociation of excitons to free holes and electrons at room temperature; ${ }^{4-9}$ (c) small electron and hole effective masses $\left(<0.5 m_{\mathrm{e}}, m_{\mathrm{e}}\right.$ is the free electron mass) along two different crystallographic directions for the good charge carrier transport throughout the crystal lattice together with the low possible electron/hole recombination rate on the surface; ${ }^{4,10,11}$ (d) lower valence band maxima (VBM) energy position than the $\mathrm{O}_{2} / \mathrm{H}_{2} \mathrm{O}$ level and higher conduction band minima (CBM) than the $\mathrm{H}^{+} / \mathrm{H}_{2}$ level for driving the generated holes and excited electrons for water oxidation and protons reduction, respectively. $^{12}$
Modern density functional theory (DFT) is considered nowadays as an extremely valuable tool to calculate these challenging fundamental parameters because of the encountered difficulty in obtaining or quantifying them in experiments. Achieving accurate computations, as requested here, is dependent on the type of a functional adopted for the description of different interactions associated with electronelectron correlation and exchange.

In recent computational works reported on electronic structure features of three-dimensional transition-metal oxide-, oxynitride-, and nitride-based semiconductor materials, ${ }^{13-20}$ we have demonstrated that the use of DFT together with the range-separated hybrid Heyd-Scuseria-Ernzerhof (HSE06) $)^{21,22}$ exchange-correlation functional leads to much more accurate results than those obtained from the standard Perdew-Burke-Ernzerhof $(\mathrm{PBE})^{23}$ functional in comparison with experiments.

Our theoretical results obtained in these studies motivated us to carry out a systematic DFT investigation [including the perturbation approach density functional perturbation theory (DFPT)] on essential fundamental parameters for solar energy conversion of a series of large, medium, and small band gap selected (covalent, binary and ternary) materials widely

Received: October 19, 2018

Accepted: December 13, 2018

Published: December 24, 2018 
utilized in fuel cells, photocatalysis, optoelectronics, photovoltaics, and dye-sensitized solar devices such as $\mathrm{BN}, \mathrm{AlN}, \mathrm{C}$, $\mathrm{ZrO}_{2}, \mathrm{Na}_{2} \mathrm{Ta}_{4} \mathrm{O}_{11}, \mathrm{Bi}_{4} \mathrm{Ti}_{3} \mathrm{O}_{12}, \mathrm{ZnS}, \mathrm{GaN}, \mathrm{SrTiO}_{3}, \mathrm{TiO}_{2}$, $\mathrm{Bi}_{12} \mathrm{TiO}_{20}, \mathrm{SiC}, \mathrm{WO}_{3}, \mathrm{TaON}, \mathrm{ZnSe}, \mathrm{BiVO}_{4}, \mathrm{CuNbO}_{3}, \mathrm{CdS}$, AlP, ZnTe, GaP, $\mathrm{Cu}_{2} \mathrm{O}, \mathrm{AlAs}, \mathrm{Ta}_{3} \mathrm{~N}_{5}, \mathrm{BP}, \mathrm{CdSe}, \mathrm{SnWO}_{4}, \mathrm{GaAs}$, $\mathrm{CdTe}$, and $\mathrm{Si}$. In this paper, we first report the electronic band gaps of these materials by considering the spin-orbit-coupling (SOC) interaction to determine the influence of relativistic effects on the computed band gap of solids containing heavy elements (atomic number greater than 56 such as $\mathrm{Bi}, \mathrm{W}$, and $\mathrm{Ta}$ ) using the standard PBE and the range-separated hybrid HSE06 functionals. Then, we report the VBM/CBM energy levels relative to water redox potentials, the macroscopic (electronic + ionic) dielectric constants, the charge carrier effective masses, and the exciton binding energy of some relevant materials among them using both the PBE and HSE06 functionals. We systematically compared our calculated values with the available experimental data to define an accurate firstprinciple quantum approach to be followed for predicting new suitable materials for visible light-driven photochemical water splitting and helping the experimentalists with a rational design of promising candidates for solar energy conversion applications.

\section{RESULTS AND DISCUSSION}

2.1. Electronic Properties. We summarize in Figure 1 the computed band gaps with the PBE and HSE06 functionals in

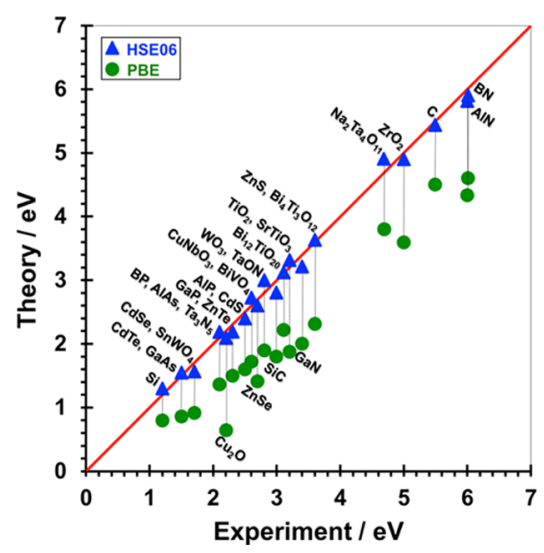

Figure 1. Small (in the $1-2 \mathrm{eV}$ range), medium (in the $2-3 \mathrm{eV}$ range), and large (in the $3-6 \mathrm{eV}$ range) band gaps of a large number of selected semiconductor crystals computed using the PBE and HSE06 functionals and compared to available experimental data. ${ }^{4,11,13-18,24-31}$

comparison with available experimental data ${ }^{4,11,13-18,24-31}$ of a series of 30 selected semiconductors such as $\mathrm{BN}, \mathrm{AlN}, \mathrm{C}, \mathrm{ZrO}_{2}$, $\mathrm{Na}_{2} \mathrm{Ta}_{4} \mathrm{O}_{11}, \mathrm{Bi}_{4} \mathrm{Ti}_{3} \mathrm{O}_{12}, \mathrm{ZnS}, \mathrm{GaN}, \mathrm{SrTiO}_{3}, \mathrm{TiO}_{2}, \mathrm{Bi}_{12} \mathrm{TiO}_{20}$, $\mathrm{SiC}, \mathrm{WO}_{3}, \mathrm{TaON}, \mathrm{ZnSe}, \mathrm{BiVO}_{4}, \mathrm{CuNbO}_{3}, \mathrm{CdS}, \mathrm{AlP}, \mathrm{ZnTe}$, $\mathrm{GaP}, \mathrm{Cu}_{2} \mathrm{O}, \mathrm{AlAs}, \mathrm{Ta}_{3} \mathrm{~N}_{5}, \mathrm{BP}, \mathrm{CdSe}, \mathrm{SnWO}_{4}, \mathrm{GaAs}, \mathrm{CdTe}$, and Si. Our obtained values with HSE06 show an excellent agreement compared to experiments with small error percentages in the $0-7 \%$ range for large or small band gaps and the 3-6\% range for medium band gaps, while those computed with PBE reveal strongly underestimated values by $18-40 \%$ for large band gaps, $32-72 \%$ for medium band gaps, and $33-47 \%$ for large band gaps with respect to the experimental ones. Detailed information about the corresponding supercell models and computed values is reported in Table
S1 in the Supporting Information. As previously reported on reference materials, ${ }^{13-20,32-35}$ the well-known band gap underestimation using generalized gradient approximation functionals can be greatly improved by the use of HSE06. The PBE functional gives smaller band gap energy values than the experimental ones, whereas the hybrid functionals such as PBE0 and B3LYP compute larger band gaps compared to experiments. ${ }^{32,33}$ These results confirm again the need of using the range-separated hybrid HSE06 functional rather than the standard one like PBE to accurately predict the experimental band gaps of semiconductor compounds.

2.2. Redox Properties. We show in Figure 2 the computed VBM and CBM energy levels using HSE06 in

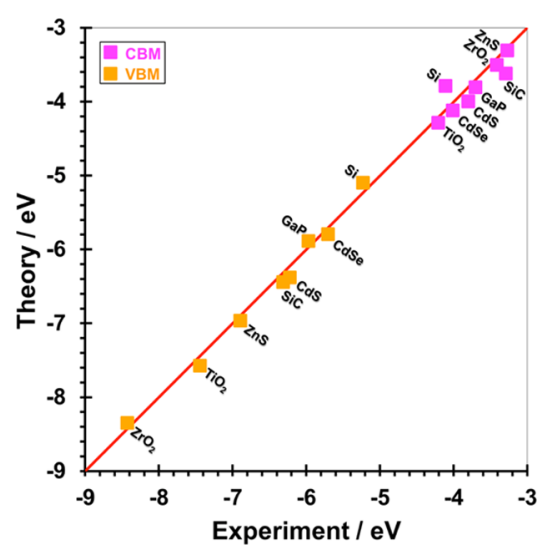

Figure 2. VBM and CBM energy levels (in eV) of $\mathrm{ZrO}_{2}, \mathrm{ZnS}, \mathrm{TiO}_{2}$, $\mathrm{SiC}, \mathrm{CdS}, \mathrm{GaP}, \mathrm{CdSe}$, and Si crystals computed using HSE06 and compared to available experimental data. ${ }^{1,26}$

comparison with available experimental data ${ }^{1,26}$ of eight selected semiconductors largely utilized in fuel cells, photocatalysis, optoelectronics, photovoltaics, and dye-sensitized solar devices, such as $\mathrm{ZrO}_{2}, \mathrm{ZnS}, \mathrm{TiO}_{2}, \mathrm{SiC}, \mathrm{CdS}, \mathrm{GaP}, \mathrm{CdSe}$, and $\mathrm{Si}$. Overall, our obtained values show a very good agreement compared to experiments with small error percentages in the $0.5-2.5 \%$ range for the VBM and $0.5-8 \%$ for the CBM. The lower accuracy for the CBM computed values with respect to the measured ones particularly in the cases of $\mathrm{SiC}$ and $\mathrm{Si}$ crystals comes from such discrepancy in the predicted band gap energy value, while the predicted VBM values were not affected that much by such a band gap difference. Detailed information about the corresponding slab models and computed values is given in Figures S1-S3 in the Supporting Information.

By applying the computational protocol based on the HSE06 functional, we have predicted suitable band gaps and VBM/CBM energy positions of $\mathrm{Ti}_{0.94} \mathrm{O}_{2} \mathrm{Se}_{0.06}, \mathrm{TiO}_{2} \mathrm{Se}_{0.06}$, $\mathrm{TiO}_{1.94} \mathrm{Se}_{0.06}, \mathrm{TiO}_{1.94} \mathrm{Te}_{0.06}, \mathrm{Ti}_{0.94} \mathrm{O}_{2} \mathrm{Te}_{0.06}, \mathrm{Ta}_{0.5} \mathrm{Nb}_{0.5} \mathrm{ON}$, and $\mathrm{Ta}_{0.75} \mathrm{~V}_{0.25} \mathrm{ON}$ materials for solar-driven photochemical water splitting (see Figure 3). $\mathrm{TiO}_{1.94} \mathrm{Se}_{0.06}, \mathrm{TiO}_{1.94} \mathrm{Te}_{0.06}$, $\mathrm{Ti}_{0.94} \mathrm{O}_{2} \mathrm{Se}_{0.06}, \mathrm{Ti}_{0.94} \mathrm{O}_{2} \mathrm{Te}_{0.06}$, and $\mathrm{TiO}_{2} \mathrm{Se}_{0.06}$ doped materials were generated from 6 at. \% of Se and Te locally substituted at $\mathrm{O}$ and $\mathrm{Ti}$ sites in the tetragonal lattice of anatase $\mathrm{TiO}_{2}$ or inserted into the anatase $\mathrm{TiO}_{2}$ crystal lattice. $\mathrm{Ta}_{0.5} \mathrm{Nb}_{0.5} \mathrm{ON}$ and $\mathrm{Ta}_{0.75} \mathrm{~V}_{0.25} \mathrm{ON}$ solid solution materials were generated from homogenous distributions of 50 at. \% of $\mathrm{Nb}$ and 25 at. \% of $\mathrm{V}$ substituted at $\mathrm{Ta}$ sites in the $\mathrm{TaON}$ monoclinic crystal lattice, respectively. Detailed information about the corresponding 


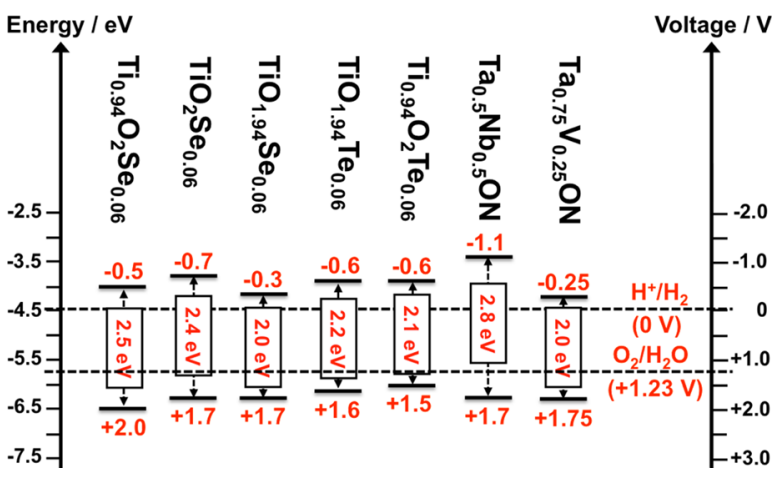

Figure 3. Predicted band gaps (in eV) and VBM/CBM levels (in eV vs vacuum or $\mathrm{V}$ vs NHE) using $\mathrm{HSE06}$ of $\mathrm{Ti}_{0.94} \mathrm{O}_{2} \mathrm{Se}_{0.06}, \mathrm{TiO}_{2} \mathrm{Se}_{0.06}$, $\mathrm{TiO}_{1.94} \mathrm{Se}_{0.06}, \mathrm{TiO}_{1.94} \mathrm{Te}_{0.06}, \mathrm{Ti}_{0.94} \mathrm{O}_{2} \mathrm{Te}_{0.06}, \mathrm{Ta}_{0.5} \mathrm{Nb}_{0.5} \mathrm{ON}$, and $\mathrm{Ta}_{0.75} \mathrm{~V}_{0.25} \mathrm{ON}$ crystals for solar-driven photochemical water splitting.

DFT-based atomic and electronic structures is reported in refs. ${ }^{19,36-38}$

2.3. Dielectric and Transport Properties. Obtaining suitable band gap and VBM/CBM energy levels for $\mathrm{H}^{+}$ reduction and water oxidation cannot guarantee whether such a photocatalyst is a good candidate for solar-driven water splitting. Also, it is reasonable to note that the band positions are not a direct evidence for water splitting and modeling catalysis is more direct. As mentioned in the introduction, other fundamental properties related to the capability of exciton separation into free carriers as well as the possibility of their migration from bulk to surface need to be carefully investigated. Following this, we have first performed benchmark tests on six selected semiconductors largely utilized in photocatalysis and photovoltaics, such as $\mathrm{TiO}_{2}, \mathrm{CdS}, \mathrm{CdSe}$, GaAs, CdTe, and Si by computing their dielectric constant, electron/hole effective masses, and binding energy of exciton using PBE and HSE06 functionals and comparing them to the available experimental data. ${ }^{4-11,39}$ With respect to the dielectric constant calculations, our obtained values with HSE06 correctly reproduce the experiments with small error percentages going from 0 up to $11 \%$, while those obtained with PBE provide an overestimation by 5-33\% with respect to the measured values. The good description of a dielectric constant with HSE06 is directly dependent on the good band gap description, while the overestimation in the case of $\mathrm{PBE}$ is mainly due to the underestimated predicted band gap values compared to experiments. Regarding the charge carrier effective mass calculations, although our obtained values of hole effective masses with both PBE and HSE06 functionals give a similar trend in most of cases with error percentages going from 2 to $35 \%$ and from 0 to $29 \%$ relative to experiments, respectively, those for electron effective masses computed with HSE06 show a better accuracy in some cases compared to the measured ones with smaller error percentages in the 10-27\% range than those obtained with PBE (11$56 \%)$. Concerning the exciton binding energy calculations, our computed values with HSE06 reveal a very good agreement compared to experiments with relatively small error percentages going from 0 up to $14 \%$, whereas those calculated with PBE give strongly underestimated values by $12-56 \%$. These results clearly confirm the necessity of going beyond the standard PBE functional and using the hybrid one like HSE06 to predict with better accuracy the experimental optoelectronic properties of semiconductor materials. Detailed information about the computed values is provided in Tables S2-S4 in the Supporting Information.

By applying the HSE06-based computational scheme, we have investigated the key fundamental properties of $\mathrm{Ti}_{0.94} \mathrm{O}_{2} \mathrm{Se}_{0.06}, \quad \mathrm{TiO}_{2} \mathrm{Se}_{0.06}, \quad \mathrm{TiO}_{1.94} \mathrm{Se}_{0.06}, \mathrm{TiO}_{1.94} \mathrm{Te}_{0.06}$, $\mathrm{Ti}_{0.94} \mathrm{O}_{2} \mathrm{Te}_{0.06}, \mathrm{Ta}_{0.5} \mathrm{Nb}_{0.5} \mathrm{ON}$, and $\mathrm{Ta}_{0.75} \mathrm{~V}_{0.25} \mathrm{ON}$ crystals for solar-driven photochemical water splitting. Regarding the dielectric constant calculations, we found small values (in the 6.8-9.7 range) for $\mathrm{Ti}_{0.94} \mathrm{O}_{2} \mathrm{Se}_{0.06}, \mathrm{TiO}_{2} \mathrm{Se}_{0.06}, \mathrm{TiO}_{1.94} \mathrm{Se}_{0.06}$, $\mathrm{TiO}_{1.94} \mathrm{Te}_{0.06}$, and $\mathrm{Ti}_{0.94} \mathrm{O}_{2} \mathrm{Te}_{0.06}$, while large values of 28.5 for $\mathrm{Ta}_{0.5} \mathrm{Nb}_{0.5} \mathrm{ON}$ and 32.6 for $\mathrm{Ta}_{0.75} \mathrm{~V}_{0.25} \mathrm{ON}$ were found, respectively. This trend indicates good separation ability for excitons to free carriers using $\mathrm{Ta}_{0.5} \mathrm{Nb}_{0.5} \mathrm{ON}$ and $\mathrm{Ta}_{0.75} \mathrm{~V}_{0.25} \mathrm{ON}$, whereas this ability is expected to be low in the cases of $\mathrm{Ti}_{0.94} \mathrm{O}_{2} \mathrm{Se}_{0.06}, \quad \mathrm{TiO}_{2} \mathrm{Se}_{0.06}, \mathrm{TiO}_{1.94} \mathrm{Se}_{0.06}, \mathrm{TiO}_{1.94} \mathrm{Te}_{0.06}$, and $\mathrm{Ti}_{0.94} \mathrm{O}_{2} \mathrm{Te}_{0.06}$. Concerning the charge carrier effective mass calculations, the anisotropic nature was revealed in the obtained values of the various crystals along the three main crystallographic directions. For $\mathrm{Ti}_{0.94} \mathrm{O}_{2} \mathrm{Se}_{0.06}, \mathrm{TiO}_{2} \mathrm{Se}_{0.06}$, $\mathrm{TiO}_{1.94} \mathrm{Se}_{0.06}, \mathrm{TiO}_{1.94} \mathrm{Te}_{0.06}$, and $\mathrm{Ti}_{0.94} \mathrm{O}_{2} \mathrm{Te}_{0.06}$, we found very large hole effective mass values (in the $8-14 m_{\mathrm{e}}$ range), in line with the strongly localized p-orbitals nature of VBM states on $\mathrm{Se}$ - or Te-doping species and $\mathrm{O}$ species located around as shown in Figure 4. This trend is expected to limit the hole

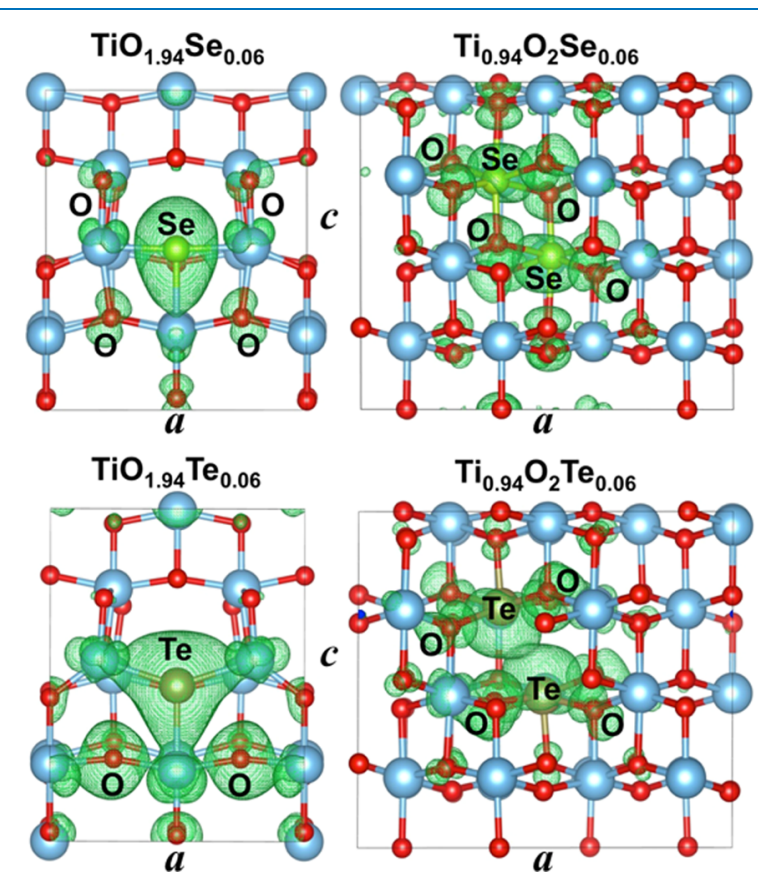

Figure 4. $2 \mathrm{D}$ illustrations over the $a c$ plane of electron density contour plots for the VBM states of $\mathrm{TiO}_{1.94} \mathrm{Se}_{0.06}, \mathrm{TiO}_{1.94} \mathrm{Te}_{0.06}$, $\mathrm{Ti}_{0.94} \mathrm{O}_{2} \mathrm{Se}_{0.06}$, and $\mathrm{Ti}_{0.94} \mathrm{O}_{2} \mathrm{Te}_{0.06}$ crystals obtained using HSE06. The isovalue is 0.004 a.u.

mobility only around the doping species, increase the electron/ hole pair recombination rate inside the bulk, and therefore, lead to low photocatalytic activities under visible-light irradiation as observed in the synthesized Se- and Te-modified $\mathrm{TiO}_{2}$ powders. ${ }^{40-42}$ As per $\mathrm{Ta}_{0.5} \mathrm{Nb}_{0.5} \mathrm{ON}$ and $\mathrm{Ta}_{0.75} \mathrm{~V}_{0.25} \mathrm{ON}$, we obtained $1.5 m_{\mathrm{e}}, 1.4 m_{\mathrm{e}}$, and $0.3 m_{\mathrm{e}}$ for holes and $0.3 m_{\mathrm{e}}$, $0.9 m_{\mathrm{e}}$, and $0.5 m_{\mathrm{e}}$ for electrons in the case of $\mathrm{Ta}_{0.5} \mathrm{Nb}_{0.5} \mathrm{ON}$ and $1.9 m_{\mathrm{e}}, 1.2 m_{\mathrm{e}}$, and $0.2 m_{\mathrm{e}}$ for holes with $1.4 m_{\mathrm{e}}, 0.4 m_{\mathrm{e}}$, and $0.7 m_{\mathrm{e}}$ for electrons in the case of $\mathrm{Ta}_{0.75} \mathrm{~V}_{0.25} \mathrm{ON}$ along the three $\langle 100\rangle,\langle 010\rangle$, and $\langle 001\rangle$ principal directions, respectively. In 
both cases, the lightest effective mass of holes is found in the $\langle 001\rangle$ orientation with values of $0.3 m_{\mathrm{e}}$ and $0.2 m_{\mathrm{e}}$, in accordance with the more pronounced delocalization nature of p-orbitals on $\mathrm{N}$ species associated to the VBM states along this specific lattice orientation (see Figure 5). The smallest

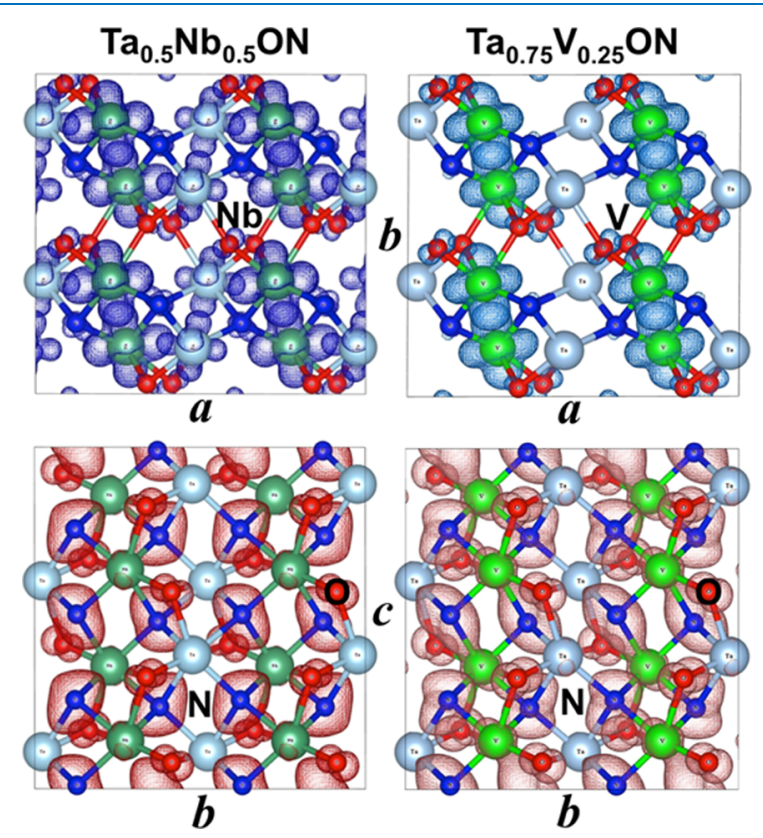

Figure 5. 2D illustrations of electron density maps over the $b c$ plane for the VBM states (on the bottom) and over the $a b$ plane for the CBM states (on the top) of $\mathrm{Ta}_{0.5} \mathrm{Nb}_{0.5} \mathrm{ON}$ and $\mathrm{Ta}_{0.75} \mathrm{~V}_{0.25} \mathrm{ON}$ crystals obtained using HSE06. The isovalue is 0.004 a.u.

electron effective mass of $0.30 m_{\mathrm{e}}$ for $\mathrm{Ta}_{0.5} \mathrm{Nb}_{0.5} \mathrm{ON}$ and $0.38 m_{\mathrm{e}}$ for $\mathrm{Ta}_{0.75} \mathrm{~V}_{0.25} \mathrm{ON}$ were obtained in the $\langle 100\rangle$ and $\langle 010\rangle$ directions, respectively, in line with the more pronounced delocalized character of d-orbitals on $\mathrm{V}, \mathrm{Nb}$, and $\mathrm{Ta}$ species corresponding to the CBM states along the two specific lattice orientations as shown in Figure 5.

As a consequence, the highest hole mobility is expected along the $\langle 001\rangle$ direction, while the highest mobility of electrons is expected either along the $\langle 100\rangle$ or $\langle 010\rangle$ orientation. Because of the fact that both effective masses are relatively small, good carrier transport features are expected along these three specific crystalline directions where the two co-catalysts for oxygen evolution reaction and hydrogen evolution reaction should be deposited on surfaces perpendicular to those crystallographic directions. Here, the holes/ electron migration seems to be easy along different crystalline orientations, and this is what we typically need to enhance the separation of holes and electrons or reduce the electron/hole recombination rate at the photocatalyst-water interface in order to enable for the holes to oxidize water and generate oxygen and for the electrons to reduce protons and generate hydrogen. Note that using this type of analysis and interpretation in the framework of combined experimental and theoretical assessments, we could successfully determine the fundamental origins behind the poor $\mathrm{H}_{2}$ or $\mathrm{O}_{2}$ evolution performance of $\mathrm{UV}$ light-responsive $\mathrm{Bi}_{12} \mathrm{TiO}_{20}$ and $\mathrm{Bi}_{4} \mathrm{Ti}_{3} \mathrm{O}_{12}$ photocatalysts as well as of common visible light-responsive $\mathrm{BiVO}_{4}, \mathrm{SnWO}_{4},\left(\mathrm{Na}_{2-x} \mathrm{Cu}_{x}\right) \mathrm{Ta}_{4} \mathrm{O}_{11}, \mathrm{TaON}$, and $\mathrm{Ta}_{3} \mathrm{~N}_{5}$ photocatalysts. ${ }^{13-18,43}$

\section{CONCLUSIONS}

Developing new semiconductor-based electronic devices for harvesting solar energy into hydrogen chemical fuel through overall water splitting reactions represents a grand opportunity at low cost for the energy future.

In the work presented here, we have investigated using DFT along with the two standard PBE and hybrid HSE06 exchange-correlation formalisms, essential fundamental parameters for solar energy conversion of a series of large, medium, and small selected (covalent, binary and ternary) materials widely utilized in fuel cells, photocatalysis, optoelectronics, photovoltaics, and dye-sensitized solar devices such as BN, AlN, C, $\mathrm{ZrO}_{2}, \mathrm{Na}_{2} \mathrm{Ta}_{4} \mathrm{O}_{11}, \mathrm{Bi}_{4} \mathrm{Ti}_{3} \mathrm{O}_{12}, \mathrm{ZnS}, \mathrm{GaN}, \mathrm{SrTiO}_{3}$, $\mathrm{TiO}_{2}, \mathrm{Bi}_{12} \mathrm{TiO}_{20}, \mathrm{SiC}, \mathrm{WO}_{3}, \mathrm{TaON}, \mathrm{ZnSe}, \mathrm{BiVO}_{4}, \mathrm{CuNbO}_{3}$, $\mathrm{CdS}$, AlP, ZnTe, GaP, $\mathrm{Cu}_{2} \mathrm{O}, \mathrm{AlAs}, \mathrm{Ta}_{3} \mathrm{~N}_{5}, \mathrm{BP}, \mathrm{CdSe}, \mathrm{SnWO}_{4}$, $\mathrm{GaAs}, \mathrm{CdTe}$, and $\mathrm{Si}$. A systematic comparison between the computed values and the available experimental data was also highlighted to confirm the accuracy of these two computational methods.

For the electronic band gap calculations, our predicted values with HSE06 showed an excellent agreement compared to experiments with small error percentages in the $0-7 \%$ range for large or small band gaps and in the 3-6\% range for medium band gaps, while those computed with PBE revealed strongly underestimated values in the $18-40 \%$ range for large band gaps, $32-72 \%$ for medium band gaps, and $33-47 \%$ for large band gaps with respect to the experimental ones. For the band energy levels relative to water redox potential calculations, our obtained values using HSE06 showed a very good agreement compared to experiments with small error percentages in the $0.5-2.5 \%$ range for $\mathrm{VBM}$ and $0.5-8 \%$ for CBM.

Regarding the dielectric constant calculations, our obtained values with HSE06 correctly reproduced the experiments with small error percentages going from 0 up to $11 \%$, whereas those obtained with PBE provided an overestimation by $5-33 \%$ with respect to the measured values. With respect to the charge carrier effective mass calculations, our obtained values of hole effective masses with both PBE and HSE06 functionals have given a similar trend in most of cases with error percentages going from 2 to $35 \%$ and from 0 to $29 \%$ relative to experiments, respectively, while those for electron effective masses computed with HSE06 showed a better accuracy in some cases compared to the measured ones with smaller error percentages in the $10-27 \%$ range than those obtained with PBE (11-56\%). Concerning the exciton binding energy calculations, our computed values with HSE06 revealed a very good agreement compared to experiments with relatively small error percentages going from 0 up to $14 \%$, whereas those calculated with $\mathrm{PBE}$ have given strongly underestimated values in the $12-56 \%$ range.

In conclusion, we have clearly shown that the computational approach based on DFT along with the range-separated hybrid HSE06 reveals very good accuracy in predicting the optoelectronic and redox properties of semiconductors, thanks to precise electronic structure calculations. The use of this firstprinciple quantum methodology led us to provide a rational design of new suitable solid solution materials for visible lightdriven photochemical water splitting. This strategy will definitely be applied to predict and propose promising candidates to the experimentalists for solar-to-chemical energy conversion. 


\section{COMPUTATIONAL METHODS}

The simulation of the studied materials was performed by considering the common crystalline phases identified experimentally using both synchrotron and neutron powder diffraction techniques (see Table $S 1$ in the Supporting Information for more details). The corresponding crystal structures were fully optimized using the periodic DFT implemented in the Vienna $\mathrm{Ab}$ initio Software Package (VASP). ${ }^{44-47}$ As reported in Table S1, supercell models were considered rather than unit cells to better describe the required properties by minimizing any possible error that might be generated by the periodic boundaries. The PBE functional, ${ }^{23}$ cutoff energy of $400 \mathrm{eV}$ for wave functions, projector augmented-wave approach, ${ }^{48}$ and different Monkhorst-Pack $k$-point grids $^{49}$ for Brillouin zone integrations were employed, as reported in Table S1. The valence electrons treated in the computations were $2 \mathrm{~s}^{2} 2 \mathrm{p}^{1}$ for $\mathrm{B}, 2 \mathrm{~s}^{2} 2 \mathrm{p}^{3}$ for $\mathrm{N}$, $3 s^{2} 3 p^{1}$ for $\mathrm{Al}, 4 s^{2} 4 p^{1}$ for Ga, $2 s^{2} 2 p^{2}$ for C, $3 d^{10} 4 s^{2}$ for $\mathrm{Zn}$, $3 s^{2} 3 p^{4}$ for $S, 4 s^{2} 4 p^{4}$ for Se, $3 s^{2} 3 p^{3}$ for $P, 5 s^{2} 4 p^{4}$ for Te, $4 s^{2} 4 p^{3}$ for As, $4 \mathrm{~d}^{10} 5 \mathrm{~s}^{2}$ for $\mathrm{Cd}, 3 \mathrm{~s}^{2} 3 \mathrm{p}^{2}$ for $\mathrm{Si}, 4 \mathrm{~d}^{2} 5 \mathrm{~s}^{2}$ for $\mathrm{Zr}, 2 \mathrm{~s}^{2} 2 \mathrm{p}^{4}$ for $\mathrm{O}$, $3 d^{2} 4 s^{2}$ for Ti, $5 d^{4} 6 s^{2}$ for $\mathrm{W}, 5 d^{3} 6 s^{2}$ for Ta, $6 s^{2} 6 p^{3}$ for Bi, $3 d^{3} 4 s^{2}$ for $\mathrm{V}, 5 \mathrm{~s}^{2} 5 \mathrm{p}^{2}$ for $\mathrm{Sn}, 3 \mathrm{~s}^{1}$ for $\mathrm{Na}, 4 \mathrm{~s}^{2} 4 \mathrm{p}^{6} 5 \mathrm{~s}^{2}$ for $\mathrm{Sr}, 3 \mathrm{~d}^{10} 4 \mathrm{~s}^{1}$ for $\mathrm{Cu}$, and $4 \mathrm{~s}^{2} 4 \mathrm{p}^{6} 4 \mathrm{~d}^{4} 5 \mathrm{~s}^{1}$ for $\mathrm{Nb}$. The ionic positions and the lattice parameters were fully optimized until the values of the Hellman-Feynman forces were less than $0.01 \mathrm{eV} \cdot \AA^{-1}$. For the self-consistent field cycles, an energy convergence criterion of $10^{-6} \mathrm{eV}$ was fixed for each supercell. Our PBE calculated lattice constants were found in very good agreement compared to experiments with very small percentage errors going from 0 to $1 \%$. Additional runs with $500 \mathrm{eV}$ cutoff energy showed that the lattice constants and bonding lengths reached their convergence at $400 \mathrm{eV}$.

The electronic band gap and $k$-space band structure of each material were computed by employing the standard $\mathrm{PBE}^{23}$ and the range-separated hybrid HSE0 $6^{21,22}$ exchange-correlation functionals implemented in VASP program, based on the optimized geometries obtained using PBE. Note that in the HSE06 formalism, a range separation approach is counted for the exchange part, while the correlation part is defined by PBE. The range-separation parameter was fixed at $0.2 \AA$. For longrange interactions, the PBE exchange is taken, whereas a mixture with $25 \%$ of Hartree-Fock and $75 \%$ of PBE is used for short-range interactions. To determine the influence of relativistic effects on the computed band gap of solids containing heavy elements (atomic number greater than 56), the SOC interaction was also considered using both PBE and HSE06 functionals. An increased cutoff energy of $500 \mathrm{eV}$ was used to guarantee good convergence of the band gaps.

The electronic dielectric constant of each compound was calculated using the self-consistent response of the crystal to an external electric field ${ }^{50}$ implemented in the VASP program along with the PBE and HSE06 functionals. The ionic dielectric constant was obtained by calculating the phonon frequencies of the crystal by means of DFPT within the linear response method implemented in VASP using the PBE formalism. The macroscopic dielectric constant was obtained from the sum of the two electronic and ionic contributions. The effective masses for holes and electrons of each material were calculated based on their electronic band structure obtained with PBE and HSE06 using the finite difference method. ${ }^{51}$ The exciton binding energy of each compound was computed using the hydrogenic model. ${ }^{52}$ More information about these methods is reported in refs. ${ }^{13-16}$

$\mathrm{ZrO}_{2}, \mathrm{ZnS}, \mathrm{TiO}_{2}, \mathrm{SiC}, \mathrm{CdS}, \mathrm{GaP}, \mathrm{CdSe}$, and $\mathrm{Si}-$ vacuum interfaces were simulated by building $(2 \times 1),(2 \times 1),(1 \times 1)$, $(1 \times 1),(1 \times 1),(2 \times 1),(1 \times 1)$, and $(1 \times 1)$ thick slabs containing $6,8,6,14,13,8,13$, and 9 atomic layers of the most common (100), (110), (110), (110), (110), (110), (110), and (111) surfaces, respectively, as shown in Figure $S 1$ in the Supporting Information. Each slab was relaxed by keeping constant the lattice parameters at the optimized values of the bulk. The vacuum thickness was refined for each crystal to minimize such possible interactions between the two opposite borders of the slab. Besides, the slab thickness needed to obtain the bulk band gap of each material was also carefully checked. Slab and vacuum thicknesses of 15, 14, 15, 20, 20, 14, 20 , and $20 \AA$ well reproduced the bulk signature of $\mathrm{ZrO}_{2}, \mathrm{ZnS}$, $\mathrm{TiO}_{2}, \mathrm{SiC}, \mathrm{CdS}, \mathrm{GaP}, \mathrm{CdSe}$, and $\mathrm{Si}$ crystals, respectively, as shown in Figures S2 and S3 in the Supporting Information when compared to the computed bulk band gap values reported in Table S1. Note that the Si located on the upper layers of $\mathrm{Si}$ and $\mathrm{SiC}$ slabs was passivated with $\mathrm{H}$ to reproduce correctly the band gap of these materials by removing any spurious electronic states coming from unsaturated or subcoordinated Si species on the surface. The absolute energy of vacuum was obtained for each crystal from the total local potential profile calculation using the VASP program together with the HSE06 functional over plans parallel to the corresponding surfaces, as shown in Figures S2 and S3. Such errors appeared from boundary conditions were corrected in each case by including corrections on the dipole during the local potential computation. ${ }^{53}$ Then, the VBM and CBM energy positions of each crystal relative to the vacuum level were obtained from the difference between the energies of VBM and CBM obtained using the slab calculations and the energy of the vacuum level. Detailed information about this methodology is reported in ref 43 .

\section{ASSOCIATED CONTENT}

\section{Supporting Information}

The Supporting Information is available free of charge on the ACS Publications website at DOI: 10.1021/acsomega.8b02884.

Fractional coordinates of the bulk optimized structures, additional tables reporting bulk structural and optoelectronic parameters, and additional figures showing slab models with band edge energy position calculation details (PDF)

\section{AUTHOR INFORMATION}

\section{Corresponding Author}

*E-mail: moussab.harb@kaust.edu.sa. Phone: +966-0128080788 (M.H.).

ORCID ${ }^{\circ}$

Moussab Harb: 0000-0001-5540-9792

Luigi Cavallo: 0000-0002-1398-338X

Notes

The authors declare no competing financial interest.

\section{ACKNOWLEDGMENTS}

This research project was supported by King Abdullah University of Science and Technology (KAUST). M.H. and 
L.C. acknowledge the KAUST Supercomputing Laboratory using the supercomputer Shaheen II for providing the needed computational resources to achieve this work.

\section{REFERENCES}

(1) Kudo, A.; Miseki, Y. Heterogeneous photocatalyst materials for water splitting. Chem. Soc. Rev. 2009, 38, 253-278.

(2) Maeda, K.; Domen, K. Photocatalytic Water Splitting: Recent Progress and Future Challenges. J. Phys. Chem. Lett. 2010, 1, 26552661.

(3) Esswein, A. J.; Nocera, D. G. Hydrogen Production by Molecular Photocatalysis. Chem. Rev. 2007, 107, 4022-4047.

(4) Madelung, O. Semiconductors: Data Handbook, 3rd ed.; Springer: New York, 2004; pp 1-691.

(5) Pelant, I.; Valenta, J. Luminescence Spectroscopy of Semiconductors; Oxford University Press: Oxford, U.K., 2012, pp 1-560.

(6) Young, K. F.; Frederikse, H. P. R. Compilation of the Static Dielectric Constant of Inorganic Solids. J. Phys. Chem. Ref. Data 1973, 2, 313-410

(7) Taguchi, T.; Shirafuji, J.; Inuishi, Y. Excitonic Emission in Cadmium Telluride. Phys. Status Solidi B 1975, 68, 727-738.

(8) Gilleo, M. A.; Bailey, P. T.; Hill, D. E. Free-Carrier and Exciton Recombination Radiation in GaAs. Phys. Rev. 1968, 174, 898-905.

(9) Zhang, J. M.; Ruf, T.; Lauck, R.; Cardona, M. Isotope Effects on Exciton Energies in CdS. Phys. Rev. B: Condens. Matter Mater. Phys. 1998, 57, 9716-9722.

(10) Adashi, S. GaAs and Related Materials; World Scientific Publishing Co. Pvt. Ltd.: Singapore, 1994.

(11) Vurgaftman, I.; Meyer, J. R.; Ram-Mohan, L. R. Band parameters for III-V compound semiconductors and their alloys. J. Appl. Phys. 2001, 89, 5815-5875.

(12) Linsebigler, A. L.; Lu, G.; Yates, J. T. Photocatalysis on $\mathrm{TiO}_{2}$ Surfaces: Principles, Mechanisms, and Selected Results. Chem. Rev. 1995, 95, 735-758.

(13) Lardhi, S.; Cavallo, L.; Harb, M. Determination of the Intrinsic Defect at the Origin of Poor $\mathrm{H}_{2}$ Evolution Performance of the Monoclinic $\mathrm{BiVO}_{4}$ Photocatalyst Using Density Functional Theory. J. Phys. Chem. C 2018, 122, 18204-18211.

(14) Lardhi, S.; Noureldine, D.; Harb, M.; Ziani, A.; Cavallo, L.; Takanabe, K. Determination of the electronic, dielectric, and optical properties of sillenite $\mathrm{Bi}_{12} \mathrm{TiO}_{20}$ and perovskite-like $\mathrm{Bi}_{4} \mathrm{Ti}_{3} \mathrm{O}_{12}$ materials from hybrid first-principle calculations. J. Chem. Phys. 2016, 144, 134702

(15) Harb, M.; Ziani, A.; Takanabe, K. Critical difference between optoelectronic properties of $\alpha$ - and $\beta$-SnWO ${ }_{4}$ semiconductors: A DFT/HSE06 and experimental investigation. Phys. Status Solidi B 2016, 253, 1115-1119.

(16) Nurlaela, E.; Harb, M.; Del Gobbo, S.; Vashishta, M.; Takanabe, K. Combined Experimental and Theoretical Assessments of the Lattice Dynamics and Optoelectronics of $\mathrm{TaON}$ and $\mathrm{Ta}_{3} \mathrm{~N}_{5} . J$. Solid State Chem. 2015, 229, 219-227.

(17) Nurlaela, E.; Ould-Chikh, S.; Harb, M.; del Gobbo, S.; Aouine, M.; Puzenat, E.; Sautet, P.; Domen, K.; Basset, J.-M.; Takanabe, K. Critical Role of the Semiconductor-Electrolyte Interface in Photocatalytic Performance for Water-Splitting Reactions Using $\mathrm{Ta}_{3} \mathrm{~N}_{5}$ Particles. Chem. Mater. 2014, 26, 4812-4825.

(18) Harb, M.; Masih, D.; Ould-Chikh, S.; Sautet, P.; Basset, J.-M.; Takanabe, K. Determination of the Electronic Structure and UV-Vis Absorption Properties of $\left(\mathrm{Na}_{2-\mathrm{x}} \mathrm{Cu}_{\mathrm{x}}\right) \mathrm{Ta}_{4} \mathrm{O}_{11}$ from First-Principle Calculations. J. Phys. Chem. C 2013, 117, 17477-17484.

(19) Harb, M. New Insights into the Origin of Visible-Light Photocatalytic Activity in Se-Modified Anatase $\mathrm{TiO}_{2}$ from Screened Coulomb Hybrid DFT Calculations. J. Phys. Chem. C 2013, 117, 25229-25235.

(20) Harb, M.; Sautet, P.; Raybaud, P. Origin of the Enhanced Visible-Light Absorption in N-Doped Bulk Anatase $\mathrm{TiO}_{2}$ from FirstPrinciples Calculations. J. Phys. Chem. C 2011, 115, 19394-19404.
(21) Heyd, J.; Scuseria, G. E.; Ernzerhof, M. Hybrid Functionals Based on a Screened Coulomb Potential. J. Chem. Phys. 2003, 118, $8207-8215$

(22) Heyd, J.; Scuseria, G. E.; Ernzerhof, M. Erratum: "Hybrid functionals based on a screened Coulomb potential" [J. Chem. Phys. 118, 8207 (2003)]. J. Chem. Phys. 2006, 124, 219906.

(23) Perdew, J. P.; Burke, K.; Ernzerhof, M. Generalized Gradient Approximation Made Simple. Phys. Rev. Lett. 1996, 77, 3865-3868.

(24) Cassabois, G.; Valvin, P.; Gil, B. Hexagonal Boron Nitride is an Indirect Bandgap Semiconductor. Nat. Photonics 2016, 10, 262-266.

(25) Feneberg, M.; Leute, R. A. R.; Neuschl, B.; Thonke, K.; Bickermann, M. High-Excitation and High-Resolution Photoluminescence spectra of Bulk AlN. Phys. Rev. B: Condens. Matter Mater. Phys. 2010, 82, 075208.

(26) Serpone, N.; Pelizzetti, E. Photocatalysis: Fundamentals and Applications; Wiley: New York, 1989.

(27) Rujkorakarn, R.; Nelson, A. J. Optical properties of $\mathrm{ZnS}_{1-\mathrm{x}} \mathrm{Se}_{\mathrm{x}}$ alloys fabricated by plasma-induced isoelectronic substitution. J. Appl. Phys. 2000, 87, 8557-8560.

(28) Yamazoe, S.; Yanagimoto, S.; Wada, T. Wide Band Gap and ptype Conductive $\mathrm{Cu}-\mathrm{Nb}-\mathrm{O}$ Films. Phys. Status Solidi RRL 2011, 5, $153-155$.

(29) Berger, L. I. Semiconductor Materials; CRC Press, 1996; pp 125.

(30) Haynes, W. M. CRC Handbook of Chemistry and Physics, 92nd ed.; CRC Press, 2011; pp 12-85.

(31) Meyer, B. K.; Polity, A.; Reppin, D.; Becker, M.; Hering, P.; Klar, P. J.; Sander, T.; Reindl, C.; Benz, J.; Eickhoff, M.; et al. Binary Copper Oxide Semiconductors: From Materials Towards Devices. Phys. Status Solidi B 2012, 249, 1487-1509.

(32) Le Bahers, T.; Rérat, M.; Sautet, P. Semiconductors Used in Photovoltaic and Photocatalytic Devices: Assessing Fundamental Properties from DFT. J. Phys. Chem. C 2014, 118, 5997-6008.

(33) Paier, J.; Marsman, M.; Hummer, K.; Kresse, G.; Gerber, I. C.; Ángyán, J. G. Screened hybrid density functionals applied to solids. J. Chem. Phys. 2006, 124, 154709.

(34) Hafner, J. Ab-initiosimulations of materials using VASP: Density-functional theory and beyond. J. Comput. Chem. 2008, 29, 2044-2078.

(35) Crowley, J. M.; Tahir-Kheli, J.; Goddard, W. A., III Resolution of the Band Gap Prediction Problem for Materials Design. J. Phys. Chem. Lett. 2016, 7, 1198-1203.

(36) Harb, M. Screened Coulomb Hybrid DFT Study on Electronic Structure and Optical Properties of Anionic and Cationic Te-Doped Anatase $\mathrm{TiO}_{2}$. J. Phys. Chem. C 2013, 117, 12942-12948.

(37) Harb, M. First-Principles Investigation of Optoelectronic and Redox Properties of $\left(\mathrm{Ta}_{1-\mathrm{x}} \mathrm{Nb}_{\mathrm{x}}\right) \mathrm{ON}$ Compounds for Photocatalysis. J. Phys. Chem. C 2015, 119, 4565-4572.

(38) Harb, M.; Cavallo, L. Suitable Fundamental Properties of $\mathrm{Ta}_{0.75} \mathrm{~V}_{0.25} \mathrm{ON}$ Material for Visible-Light-Driven Photocatalysis: A DFT Study. ACS Omega 2016, 1, 1041-1048.

(39) Gonzalez, R. J.; Zallen, R.; Berger, H. Infrared reflectivity and lattice fundamentals in anatase $\mathrm{TiO}_{2}$ s. Phys. Rev. B: Condens. Matter Mater. Phys. 1997, 55, 7014-7017.

(40) Rockafellow, E. M.; Haywood, J. M.; Witte, T.; Houk, R. S.; Jenks, W. S. Selenium-Modified TiO2and Its Impact on Photocatalysis. Langmuir 2010, 26, 19052-19059.

(41) Stengl, V.; Bakardjieva, S.; Bludská, J. Se and Te-Modified Titania for Photocatalytic Applications. J. Mater. Sci. 2011, 46, 35233536.

(42) Padmalatha, P.; Khatri, P. K.; Jain, S. L. Selenium-Doped $\mathrm{TiO}_{2}$ as an Efficient Photocatalyst for the Oxidation of Tetrahydrofuran to $\gamma$-Butyrolactone Using Hydrogen Peroxide as Oxidant. Synlett 2013, 24, 1405-1409.

(43) Harb, M.; Sautet, P.; Nurlaela, E.; Raybaud, P.; Cavallo, L.; Domen, K.; Basset, J.-M.; Takanabe, K. Tuning the Properties of Visible-Light-Responsive Tantalum (Oxy)Nitride Photocatalysts by Non-Stoichiometric Compositions: A First-Principles Viewpoint. Phys. Chem. Chem. Phys. 2014, 16, 20548-20560. 
(44) Kresse, G.; Hafner, J. Ab initiomolecular-dynamics simulation of the liquid-metal-amorphous-semiconductor transition in germanium. Phys. Rev. B: Condens. Matter Mater. Phys. 1994, 49, 1425114269 .

(45) Kresse, G.; Furthmüller, J. Efficient iterative schemes forab initiototal-energy calculations using a plane-wave basis set. Phys. Rev. B: Condens. Matter Mater. Phys. 1996, 54, 11169-11186.

(46) Kresse, G.; Furthmüller, J. Efficiency of Ab-Initio Total Energy calculations for Metals and Semiconductors Using a Plane-Wave Basis Set. Comput. Mater. Sci. 1996, 6, 15-50.

(47) Kresse, G.; Joubert, D. From Ultrasoft Pseudopotentials to the Projector Augmented-Wave Method. Phys. Rev. B: Condens. Matter Mater. Phys. 1999, 59, 1758-1775.

(48) Blöchl, P. E. Projector Augmented-Wave Method. Phys. Rev. B: Condens. Matter Mater. Phys. 1994, 50, 17953-17979.

(49) Monkhorst, H. J.; Pack, J. D. Special points for Brillouin-zone integrations. Phys. Rev. B: Solid State 1976, 13, 5188-5192.

(50) Souza, I.; Íñiguez, J.; Vanderbilt, D. First-Principles Approach to Insulators in Finite Electric Fields. Phys. Rev. Lett. 2002, 89, 117602.

(51) Fonari, A.; Sutton, C. Effective Mass Calculator for Semiconductors. http://afonari.com/emc/ (accessed April 05, 2018).

(52) Rodina, A. V.; Dietrich, M.; Göldner, A.; Eckey, L.; Hoffmann, A.; Efros, A.; Rosen, M.; Meyer, B. K. Free Excitons in Wurtzite GaN. Phys. Rev. B: Condens. Matter Mater. Phys. 2001, 64, 115204.

(53) Makov, G.; Payne, M. C. Periodic boundary conditions inab initiocalculations. Phys. Rev. B: Condens. Matter Mater. Phys. 1995, 51, 4014-4022. 